\title{
Lattice gauge theory with staggered fermions: how, where, and why (not)
}

\section{Andreas S. Kronfeld*}

Theoretical Physics Department, Fermi National Accelerator Laboratory, ${ }^{\dagger}$ Batavia, Illinois, USA

E-mail: ask@fnal.gov

Many results from lattice QCD of broad importance to particle and nuclear physics are obtained with $2+1$ flavors of staggered sea quarks. In the continuum limit, staggered fermions yield four species, called tastes. To reduce the number of tastes to one (per flavor), the simulation employs the fourth root of the four-taste staggered fermion determinant. This talk surveys evidence in favor of this procedure, refutes recent criticisms, and reviews recent algorithmic and technical improvements. Physics results are covered in other plenary talks.

The XXV International Symposium on Lattice Field Theory

July 30-4 August 2007

Regensburg, Germany

\footnotetext{
* Speaker.

$\dagger$ Operated by Fermi Research Alliance, LLC, under Contract No. DE-AC02-07CH11359 with the United States Department of Energy.
} 


\section{Why this paper?}

Lately lattice QCD has enjoyed considerable success. In 2003 the HPQCD, MILC, and Fermilab Lattice Collaborations found that calculations based on Monte Carlo simulations agreed with experiment at the 1-3\% level for a wide variety of physical quantities [1]. During the next two years the same techniques were used to predict the shape of the $D \rightarrow K l v$ semileptonic form factor [2], the mass of the $B_{c}$ meson [3], and the decay constants of the $D$ and $D_{s}$ mesons [4]. Each of these results was subsequently confirmed by experimental measurements [5]. Lattice QCD was also used to determine the strong coupling $\alpha_{s}$ [6]. These results have made a strong impression in the fields of particle physics and nuclear physics, and a long-standing claim of lattice gauge theorists is now generally accepted: the combination of numerical simulation [7] and chiral perturbation theory [8] is a sound way to solve gauge theories, such as QCD.

The key to this development was the incorporation of $2+1$ flavors of sea quarks with the fastest technique, "improved staggered fermions with the Asqtad action" [9]. Unsurprisingly for computational science, the fastest technique has some unresolved theoretical questions hanging over it. This state of affairs presents a great opportunity. It is simple common sense to reproduce the results of Refs. $[1,2,3,4,6]$ with theoretically cleaner treatments of sea quarks. Such methods require more computation, so one can argue (persuasively) to devote more computer resources to lattice QCD than in the past. At the same time, there is a strong motivation (and obligation) to understand theoretically how, where, and why staggered fermions work so well.

The crux of the unresolved issues is the fermion doubling problem. Staggered fermions cope with the problem partially, reducing the number of species from sixteen (in four space-time dimensions) to four. The empirically successful results cited above all use ensembles generated and made publicly available by the MILC Collaboration [10]. To reduce the number of fermion species (per flavor) from four to one, gauge fields in these ensembles have the partition function [11]

$$
Z=\int \mathscr{D} U \prod_{q}\left[\operatorname{det}_{4}\left(\not D_{\text {stag }}+m_{q}\right)\right]^{1 / 4} e^{-S_{\text {gauge }}}
$$

where $S_{\text {gauge }}$ is the action for the gluons, $\not_{\text {stag }}$ is the (improved) staggered discretization of the Dirac operator, and the subscript 4 on the determinant emphasizes the underlying number of species. The question, then, is whether $\left[\operatorname{det}_{4}\left(\not_{\text {stag }}+m_{q}\right)\right]^{1 / 4}$ is legitimate.

This so-called "rooting procedure" is controversial. Critics seem to accept that Eq. (1.1) is valid in perturbation theory, where internal fermion loops are simply multiplied by $\frac{1}{4}$. Several interested and disinterested parties are investigating non-perturbative aspects, sometimes finding undesirable features though not, to my knowledge, fatal flaws. An apparent exception is the work of Michael Creutz [12, 13, 14, 15], including these Proceedings [16], which does claim that rooting fails. Although these papers have already been refuted [17], the organizers of Lattice 2007 invited me to respond to Creutz's claims "on behalf of the staggered community." My charge is to "increase the confidence of the wider lattice community in the rooting procedure" (italics added).

The organizers also asked me to comment on the relevance of lattice gauge theory to CERN's Large Hadron Collider (LHC). This request and the need to anticipate new critiques have shaped the organization of this talk. Section 2 is an essay presenting the view that the LHC era will require fast lattice calculations and, thus, a broader understanding of validity of the rooting procedure. Sections 3 and 4 disentangle the main issues. Even without rooting, staggered fermions bring 
in complications, which are reviewed in sect. 3, focusing on a new quantum number "taste" that characterizes the remaining species doubling. Furthermore, even with full SU( $\left.4 n_{f}\right)$ taste symmetry, rooting has its peculiarities, which may be unfamiliar but should not be controversial; they are explained in sect. 4. These two strands are then brought together in sect. 5 to discuss rooted staggered fermions. It is then possible to refute Refs. [12, 13, 14, 15, 16] relatively tersely, in sect. 6. Section 7 covers some new developments, and sect. 8 offers a summary and perspective.

\section{The lattice community in the LHC era}

To represent the "staggered community" before the "wider lattice community" it helps to think about who belongs to each of them. The wider lattice community simply consists of those who attend conferences on lattice field theory and submit e-prints to the hep-lat section of the arXiv. The staggered community includes not only those who generate large ensembles of SU(3) gauge fields with rooted, staggered sea quarks, but also those (like me) who use them. The staggered community also reaches beyond hep-lat. Many physicists without much expertise in lattice gauge theory have a stake in the validity of the rooting procedure. For example, the Heavy Flavor Averaging Group [18] and similar enterprises use lattice QCD results to help them understand whether non-Standard phenomena contribute to $B$-meson decays. It is therefore useful to take the staggered community to be everyone who thinks the validity of staggered fermions is worthy of study.

The organizers' charge implies that there may be some in the wider lattice community who do not see themselves as part of the staggered community, even as defined here. That would be unfortunate. Despite the "controversial" nature of Eq. (1.1), the arguments against it are difficult to pin down, because they are not documented in the scientific literature. Mike Creutz is to be commended for writing up his critique, making it possible to decide whether his qualms are correct. On the other hand, some members of the lattice community seem to prefer sniping from the sidelines. They may hope that increases in computer resources will make the controversy moot, by allowing other methods for sea quarks to catch up. The status of other methods is surveyed in Refs. [19], and one may judge for oneself. My reading is that other methods have not caught up, partly because algorithms for the staggered sea are not standing still [20].

Future calculations are more salient than past efforts, so it is worth looking ahead to the LHC era. The focus of the LHC's physics program will be on the terascale, ${ }^{1}$ where we expect to find the agents of electroweak symmetry breaking. They may be strongly coupled and, if so, the need for numerical lattice gauge theory will skyrocket. A lot will be at stake, and the fastest way to elucidate the physics will prevail. Even if physics at the terascale is weakly-coupled or, worse, boring, a need will remain for precision and accuracy in $f_{K}, f_{B} \sqrt{B_{B}}$, etc. In addition, searches for non-Standard particles will profit from good calculations of moments of parton densities, especially the gluon density, so that signal and background cross sections can be calculated.

In summary, whether for QCD or for other gauge theories, the fastest correct technique for simulating the fermion sea will remain a key tool during the LHC era. New researchers, young and old, may turn to lattice gauge theory, and they will expect that we established experts have understood whether rooted staggered fermions are valid, or not. For this reason I think the whole

\footnotetext{
${ }^{1}$ Using the term "terascale" for the teraelectronvolt energy scales was inspired by terascale (i.e., teraflop/s and terabyte) computing (J.D. Lykken, private communication).
} 
lattice community should engage in an open-minded scientific debate, and skeptics should submit their criticisms of rooted staggered fermions to the hep-lat arXiv.

\section{Staggered fermions without rooting}

Most of the complexity of rooted staggered quarks has nothing to do with the rooting procedure. Instead, it stems from the way spacetime and flavor-like symmetries of four (or $4 n_{f}$ ) Dirac fermions emerge in the continuum limit. The aim of this section, therefore, is to give a brief review of the definitions and symmetries of staggered fermions and their connection with the continuum limit. Most of this is not rigorously proven, but it is fair to say that it is established, principally because numerical simulations shore up the theoretical picture.

The simplest discretization of fermions replaces Dirac's covariant derivative with a nearestneighbor interaction. The resulting "naïve" action is

$$
S_{\text {naïve }}=\frac{1}{2} a^{3} \sum_{x, \mu} \bar{\Upsilon}(x) \gamma_{\mu}\left[U_{\mu}(x) \Upsilon(x+\hat{\mu} a)-U_{\mu}^{\dagger}(x-\hat{\mu} a) \Upsilon(x-\hat{\mu} a)\right]+m_{0} a^{4} \sum_{x} \bar{\Upsilon}(x) \Upsilon(x),
$$

where each site $x$ possesses Grassmann variables $\Upsilon_{\alpha}^{i}$ and $\bar{\Upsilon}_{\alpha}^{i}$, with $i$ and $\alpha$ the color and spinor indices. This action is invariant under color $\mathrm{SU}\left(N_{c}\right)$, lattice translations, and hypercubic rotations. With $n_{f}$ flavors there is a $\mathrm{U}_{\mathrm{V}}\left(n_{f}\right) \times \mathrm{U}_{\mathrm{A}}\left(n_{f}\right)$ chiral flavor symmetry, softly broken by the masses.

The naïve action also possesses a remarkable SU(4) "doubling symmetry" [21], with fifteen $x$-dependent (anti-Hermitian) generators $B^{A}(x)$ :

$$
B_{\mu}(x)=\gamma_{\mu} \gamma_{5}(-1)^{n_{\mu}}, \quad B_{5}(x)=i \gamma_{5} \varepsilon(x), \quad B_{\mu}(x) B_{5}(x), \quad B_{\mu}(x) B_{v}(x) \quad(\mu<v),
$$

where $n=x / a$, and $\varepsilon(x)=(-1)^{n_{1}+n_{2}+n_{3}+n_{4}}$. The naïve fermion field transforms as

$$
\Upsilon(x) \mapsto e^{\omega^{A} B^{A}(x)} \Upsilon(x), \quad \bar{\Upsilon}(x) \mapsto \bar{\Upsilon}(x) e^{-\omega^{A} B^{A}(x)} .
$$

The physical interpretation becomes clear in momentum space. Consider, for example, a doubling transformation generated by $B_{\mu}: \Upsilon(p) \mapsto \cos \omega \Upsilon(p)+\sin \omega \gamma_{\mu} \gamma_{5} \Upsilon(p+\hat{\mu} \pi / a)$. In general, the doubling symmetries relate all 16 corners $\pi^{A} / a$ of the Brillouin zone, up to a shuffling of the Dirac index. (See Eqs. (3.16)-(3.20), below, for a complete list of the 4-vectors $\pi^{A}$.)

The physical consequence of the doubling symmetry (in four dimensions) is that a single naïve fermion field $\Upsilon$ corresponds to 16 species of fermion. The extra species are evident in vacuum polarization, leading to $\beta_{0}=\frac{11}{3} N_{c}-\frac{2}{3} 16 n_{f}$ in the running of the gauge coupling [21]. The axial anomaly receives contributions from all 16 species, in the pattern [21]

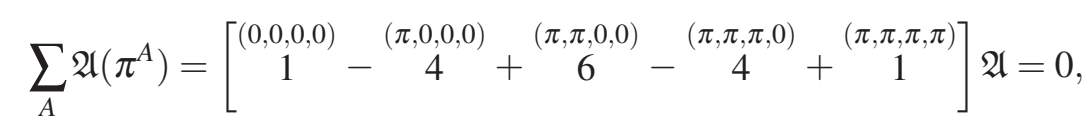

where a typical $\pi^{A}$ hovers over the integer multiplicity of the species from that kind of corner. The total anomaly vanishes because with the naïve action the flavor-singlet axial symmetry $U_{A}(1)$ is exact. Because the anomaly is wrong, naïve fermions do not seem to be what one wants for QCD.

The doubling symmetries can be rendered $x$-independent via a change of variables [22]:

$$
\Upsilon(x)=\Omega(x) X(x), \quad \bar{\Upsilon}(x)=\bar{X}(x) \Omega^{-1}(x), \quad \Omega(x)=\gamma_{1}^{n_{1}} \gamma_{2}^{n_{2}} \gamma_{3}^{n_{3}} \gamma_{4}^{n_{4}} .
$$


Rewriting the naïve action in the new fermion fields $X_{\alpha}^{i}, \bar{X}_{\alpha}^{i}$, one finds

$$
S_{\text {naïve }}=\frac{1}{2} a^{3} \sum_{x, \mu} \bar{X}(x) \eta_{\mu}(x)\left[U_{\mu}(x) X(x+\hat{\mu} a)-U_{\mu}^{\dagger}(x-\hat{\mu} a) X(x-\hat{\mu} a)\right]+m_{0} a^{4} \sum_{x} \bar{X}(x) X(x),
$$

where the signs $\eta_{\mu}(x)$ have replaced the Dirac matrices via

$$
\Omega^{-1}(x) \gamma_{\mu} \Omega(x \pm \hat{\mu} a)=(-1)^{\sum_{\rho<\mu} n_{\rho}}=: \eta_{\mu}(x) .
$$

Equation (3.6) can also be obtained by diagonalizing a maximal commuting subgroup of the doubling symmetry [23].

In Eq. (3.6) the transformed spinor index is sterile, so the number of degrees of freedom can be reduced four-fold, yielding

$$
S_{\text {stag }}=\frac{1}{2} a^{3} \sum_{x, \mu} \bar{\chi}(x) \eta_{\mu}(x)\left[U_{\mu}(x) \chi(x+\hat{\mu} a)-U_{\mu}^{\dagger}(x-\hat{\mu} a) \chi(x-\hat{\mu} a)\right]+m_{0} a^{4} \sum_{x} \bar{\chi}(x) \chi(x),
$$

where $\chi^{i}$ is a fermion field without a spinor index. A Hamiltonian formalism with one-component fermions and sign factors instead of Dirac spinors and matrices was introduced by Susskind [24], extending work in 1+1 dimensions by Banks, Kogut, and Susskind [25]. Because of the ubiquitous factors $(-1)^{n_{\mu}}$ the Euclidean formulation with $S_{\text {stag }}$ and $\chi^{i}$ is called "staggered fermions."

The projection from the big $X_{\alpha}^{i}$ to the little $\chi^{i}$ removes the SU(4) doubling symmetry, albeit in a not-so-straightforward way. All other symmetries-except color and the vector flavor symmetries—become $x$-dependent $[24,26]$. For example, consider translations

$$
t_{\mu}:\left\{\begin{array}{l}
\Upsilon(x) \mapsto \Upsilon(x+\hat{\mu} a) \Rightarrow X(x) \mapsto \zeta_{\mu}(x) \gamma_{\mu} X(x+\hat{\mu} a) \\
\bar{\Upsilon}(x) \mapsto \bar{\Upsilon}(x+\hat{\mu} a) \Rightarrow \bar{X}(x) \mapsto \zeta_{\mu}(x) \bar{X}(x+\hat{\mu} a) \gamma_{\mu}
\end{array},\right.
$$

where

$$
\zeta_{\mu}(x)=\Omega^{-1}(x) \Omega(x \pm \hat{\mu} a) \gamma_{\mu}=(-1)^{\sum_{\sigma>\mu} n_{\sigma}} .
$$

Because of the Dirac matrix in (3.9), we see that the projection from $X_{\alpha}^{i}$ to $\chi^{i}$ does not commute with lattice translational symmetry. On the other hand, a certain combination of translations and $B$ transformations called shifts does survive the projection:

$$
S_{\mu}:\left\{\begin{array}{l}
\Upsilon(x) \mapsto-i B_{\mu} B_{5} \Upsilon(x+\hat{\mu} a) \Rightarrow \chi(x) \mapsto \zeta_{\mu}(x) \chi(x+\hat{\mu} a) \\
\bar{\Upsilon}(x) \mapsto-i \bar{\Upsilon}(x+\hat{\mu} a) B_{\mu} B_{5} \Rightarrow \bar{\chi}(x) \mapsto \zeta_{\mu}(x) \bar{\chi}(x+\hat{\mu} a), \\
U_{v}(x) \mapsto U_{v}(x+\hat{\mu} a), \quad \forall v
\end{array}\right.
$$

and $X_{\alpha}$ transforms just like $\chi$. Similarly, rotations, spatial inversion, and charge conjugation become entangled with this residue of the doubling symmetries [26, 27, 28, 29, 30, 31].

Here we shall focus on issues related to the species content of staggered fermions. Acting on the fermion fields, the shifts anti-commute, $S_{\mu} S_{v}=-S_{v} S_{\mu}(v \neq \mu)$, from which it follows that the residue of the doubling symmetry is a discrete Clifford group $\Gamma_{4}$. Because shifts translate the gauge field, a single $\Gamma_{4}$ symmetry arises for any $n_{f}$. Shift symmetry has two kinds of irreducible representations (irreps)—fermionic and bosonic — with representation matrices, respectively,

$$
\begin{aligned}
& \mathfrak{D}^{(-)}\left(S_{\mu}\right)=\xi_{\mu} e^{i p_{\mu} a}, \\
& \mathfrak{D}^{(+)}\left(S_{\mu}\right)=s_{\mu}^{A} e^{i p_{\mu} a},
\end{aligned}
$$


where the physical momentum ranges over $p_{\mu} \in(-\pi / 2 a, \pi / 2 a]$. The fermionic representation is 4-dimensional, labeled by an index $t=1,2,3,4$, and the $\xi_{\mu}$ are $4 \times 4$ matrices obeying the Clifford algebra, $\left\{\xi_{\mu}, \xi_{v}\right\}=2 \delta_{\mu v}$. The sixteen 1-dimensional bosonic irreps are labeled by the corners $A$ of the Brillouin zone and the pre-factors are signs, $s_{\mu}^{A}=e^{i \pi_{\mu}^{A}}$. The index $t$ and the label $A$ denote a quantum number that, nowadays, is called taste.

A colored particle's taste, just like its momentum, is made complicated by $\mathrm{SU}\left(N_{c}\right)$ color gauge symmetry. It is enough, however, to consider color singlets, such as the mixed-action bilinear, which arises, for example, in heavy-light physics [32]. Let

$$
H_{t}^{(\Gamma)}(x)=\bar{\Psi}_{\alpha}(x) \Gamma_{\alpha \beta} \Omega_{\beta t}(x) \chi(x),
$$

where the matrix $\Omega$ reappears, now with the first index interpreted as a Dirac index, but the second index interpreted as taste index [33, 34]. Here the field $\bar{\Psi}$ represents a Wilson or Ginsparg-Wilson anti-fermion, so its spinor index has the conventional meaning. Under shift symmetry (as one can easily verify), the meson field $H_{t}^{(\Gamma)}$ transforms as

$$
S_{\mu}: H_{t}^{(\Gamma)}(x) \mapsto\left[\xi_{\mu}\right]_{t t^{\prime}} H_{t^{\prime}}^{(\Gamma)}(x+\hat{\mu} a), \quad \xi_{\mu}=\gamma_{\mu}^{T},
$$

so Eq. (3.15) gives an explicit realization of Eq. (3.12). Under rotations and spatial inversion $H_{t}^{(\Gamma)}$ transforms as a scalar, vector, or tensor, etc., dictated by $\Gamma$, up to a change of taste. Baryons with three staggered quarks also have fermionic taste [27, 35].

Staggered-staggered mesons have bosonic taste $A$. Rotations imply degeneracies in an almost obvious way, ${ }^{2}$ leading to multiplets

$$
\begin{aligned}
I & : \pi^{A} \in\{(0,0,0,0)\}, \\
V & : \pi^{A} \in\{(\pi, 0,0,0),(0, \pi, 0,0),(0,0, \pi, 0) ;(0,0,0, \pi)\}, \\
T & : \pi^{A} \in\{(\pi, \pi, 0,0),(\pi, 0, \pi, 0),(0, \pi, \pi, 0) ;(\pi, 0,0, \pi),(0, \pi, 0, \pi),(0,0, \pi, \pi)\}, \\
A & : \pi^{A} \in\{(0, \pi, \pi, \pi),(\pi, 0, \pi, \pi),(\pi, \pi, 0, \pi) ;(\pi, \pi, \pi, 0)\}, \\
P & : \pi^{A} \in\{(\pi, \pi, \pi, \pi)\} .
\end{aligned}
$$

These multiplets are called the taste-singlet $I$, the vector taste $V$, the tensor taste $T$, the axial vector taste $A$, and the pseudoscalar taste $P$. Another hadron with bosonic taste is a heavy-light baryon consisting of two staggered light quarks and a Fermilab or NRQCD heavy quark [36].

The staggered action, Eq. (3.8), inherits softly broken but otherwise exact chiral symmetries,

$$
\chi(x) \mapsto e^{\theta^{a} T^{a} \mathcal{E}(x)} \chi(x), \quad \bar{\chi}(x) \mapsto \bar{\chi}(x) e^{\theta^{a} T^{a} \mathcal{E}(x)},
$$

where $T^{a^{\dagger}}=-T^{a}$. With $n_{f}$ flavors, this is simply the $\mathrm{U}_{\mathrm{A}}\left(n_{f}\right)$ symmetry manifest in Eq. (3.1), made $x$-dependent via Eqs. (3.5). Thus, one has axial currents and pseudoscalar densities

$$
\begin{aligned}
A_{P}^{v a}(x) & =\frac{1}{2} \eta^{v}(x) \varepsilon(x)\left[\bar{\chi}(x+\hat{v} a) U_{v}^{\dagger}(x) T^{a} \chi(x)-\bar{\chi}(x) U_{v}(x) T^{a} \chi(x+\hat{v} a)\right], \\
P_{P}^{a}(x) & =\varepsilon(x) \bar{\chi}(x) T^{a} \chi(x),
\end{aligned}
$$

\footnotetext{
${ }^{2}$ Strictly speaking, the degeneracies are among irreps of the symmetry group of the transfer matrix, which entails cubic rotations, not hypercubic rotations $[29,30]$. Thus, states with $\pi_{4}^{A}=0, \pi$ need not be degenerate; hence the semicolons in Eqs. (3.17)-(3.19). In practice they turn out to be nearly degenerate [10].
} 
satisfying PCAC relations [37] (for equal renormalized masses $m)^{3}$

$$
a^{-1} \sum_{v}\left[A_{P}^{v a}(x)-A_{P}^{v a}(x-\hat{v} a)\right]=2 m P_{P}^{a}(x)+2 a^{-4} \varepsilon(x) \operatorname{tr} T^{a} .
$$

It is tempting to say that the axial anomaly does not appear in Eq. (3.24) owing to Eq. (3.4), with each contribution at quarter strength. It is better, however, to note that it is not expected, because the symmetry (3.21) is exact. The axial anomaly is also undesired. Under shifts $A_{P}^{v a}$ and $P_{P}^{a}$ transform as

$$
S_{\mu}:\left\{\begin{array}{rl}
A_{P}^{v a}(x) & \mapsto(-1)^{n_{\mu}} A_{P}^{v a}(x+\hat{\mu} a) \\
P_{P}^{a}(x) & \mapsto(-1)^{n_{\mu}} P_{P}^{a}(x+\hat{\mu} a)
\end{array}, \quad \forall \mu, v,\right.
$$

so one sees that these bilinears are not taste singlets, rather they transform under the (non-singlet) $P$ irrep. This non-singlet character of $A_{P}^{v a}$ and $P_{P}$ provides a clue that the new quantum number taste plays a key role in the physics that emerges in the continuum limit.

The change of variables (3.5) followed by the projection from $X_{\alpha}^{i}$ to $\chi^{i}$ reduces the number of species from 16 to 4 . The action can be rewritten using physically suggestive fields

$$
\psi_{\alpha t}(y)=\frac{1}{8} \sum_{r} \Omega_{\alpha t}(r) U(y, y+r) \chi(y+r), \quad \bar{\psi}_{\alpha t}(y)=\frac{1}{8} \sum_{r} \bar{\chi}(y+r) \Omega_{t \alpha}^{\dagger}(r) U(y+r, y),
$$

where $y$ labels hypercubes of size $2^{4}$ or, equivalently, sites on a coarser lattice of spacing $b=2 a$, $r$ runs over the hypercube, and $U(y, y+r)$ is parallel transport along some chosen path from $y$ to $y+r$. As in Eq. (3.14), the indices $\alpha$ and $t$ are interpreted as Dirac and taste indices. Suppressing the gauge field, the action is then rewritten [33,34]

$$
\begin{aligned}
S_{\mathrm{stag}} & =\frac{1}{2} b^{3} \sum_{y, \mu} \sum_{t=1}^{4} \bar{\psi}_{t}(y) \gamma_{\mu}\left[\psi_{t}(y+\hat{\mu} b)-\psi_{t}(y-\hat{\mu} b)\right]+m_{0} b^{4} \sum_{y} \sum_{t=1}^{4} \bar{\psi}_{t}(y) \psi_{t}(y) \\
& -\frac{1}{2} b^{3} \sum_{y, \mu} \sum_{t, t^{\prime}=1}^{4} \bar{\psi}_{t}(y) \gamma_{5}\left[\xi_{5} \xi_{\mu}\right]_{t t^{\prime}}\left[\psi_{t^{\prime}}(y+\hat{\mu} b)+\psi_{t^{\prime}}(y-\hat{\mu} b)-2 \psi_{t^{\prime}}(y)\right],
\end{aligned}
$$

which looks like a lattice field theory of four Dirac fermions, with a Wilson-like term to alleviate doublers. For perturbative gauge fields this appearance continues to hold. For example, the betafunction starts with $\beta_{0}=\frac{11}{3} N_{c}-\frac{2}{3} 4 n_{f}$ [23], and the exact $\Gamma_{4}$ part of the shift symmetry ensures that mass renormalization is taste-independent $[26,38]$.

The central conjecture of (unrooted) staggered fermions is that the picture of four (or $4 n_{f}$ ) Dirac fermions holds non-perturbatively. I say "conjecture" to mathematicians because it is not proven rigorously (and I could say the same about all formulations of lattice fermions), and to physicists because the coupling to gauge fields is based on the one-component action, Eq. (3.8). Consequently the spacetime and flavor-like symmetries are entangled, so it is not especially transparent how $\mathrm{SO}(4)$ rotational symmetry and $\mathrm{SU}_{\mathrm{V}}\left(4 n_{f}\right) \times \mathrm{SU}_{\mathrm{A}}\left(4 n_{f}\right) \times \mathrm{U}_{\mathrm{V}}(1)$ flavor-taste chiral symmetry emerge in the continuum limit.

To get an idea of the complications (see, e.g., Refs. [26, 30] for details), let us consider some important cases. The hypercubic rotations (denoted $\mathrm{SW}_{4}$ ) are embedded

$$
\mathrm{SW}_{4} \subset[\mathrm{SO}(4) \times \mathrm{SO}(4)]_{\mathrm{diag}} \subset \mathrm{SO}(4) \times \mathrm{SU}_{\mathrm{V}}(4) \subset \mathrm{SO}(4) \times \mathrm{SU}_{\mathrm{V}}\left(4 n_{f}\right)
$$

\footnotetext{
${ }^{3}$ In the flavor singlet $\operatorname{tr} T^{a} \neq 0$, but the last term vanishes in a average over a hypercube, so it is unimportant.
} 
into the diagonal subgroup of the Euclidean group and a flavor-singlet subgroup of the $\mathrm{SU}_{\mathrm{V}}\left(4 n_{f}\right)$ group. The Clifford group abstracted from the shifts and the vector flavor symmetries are embedded

$$
\Gamma_{4} \times \mathrm{SU}_{\mathrm{V}}\left(n_{f}\right) \subset \mathrm{SU}_{\mathrm{V}}\left(4 n_{f}\right)
$$

in the vector flavor-taste symmetries. The axial flavor symmetries are embedded

$$
\mathrm{U}_{\mathrm{A}}\left(n_{f}\right) \subset \mathrm{SU}_{\mathrm{A}}\left(4 n_{f}\right)
$$

in the flavor-taste non-singlet axial symmetries. For sect. 6, the embedding (3.30) along with Eqs. (3.22)-(3.25) are key. The exact chiral symmetries are not taste singlets. Therefore, they cannot have anything to do with anomalies, gauge-field topology, zero modes of the Dirac operator, or their consequences, such as 't Hooft vertices.

If the emerging picture is correct, staggered fermions must possess a bilinear corresponding in the continuum limit to the flavor- and taste-singlet axial current. Analogously to the case of Wilson fermions, this is not a Noether current, but the explicit breaking is superficially of order $a$. The anomalous current extends over a whole hypercube [23, 37]:

$$
\begin{array}{r}
A_{I}^{\mu}(x)=\frac{i}{32} \sum_{b+c=d} \eta^{\mu}(x+c) \eta_{1}(x+b) \eta_{2}(x+b) \eta_{3}(x+b) \eta_{4}(x+b)[ \\
\bar{\chi}(x+c+\hat{\mu} a) U_{\mu}^{\dagger}(x+c) \bar{U}(x+c, x+b) \chi(x+b) \\
\left.-\bar{\chi}(x+b) \bar{U}(x+b, x+c) U_{\mu}(x+c) \chi(x+c+\hat{\mu} a)\right],
\end{array}
$$

where $d=(\hat{1}+\hat{2}+\hat{3}+\hat{4}) a, \bar{U}(x+b, x+c)$ is the average of parallel transport over paths from $x+b$ to $x+c$, and the sum is over all 4-dimensional diagonals of the hypercube at $x$. Under shift symmetry $A_{I}^{\mu}$ is a taste singlet, as is the corresponding pseudoscalar density

$$
P_{I}(x)=\frac{i}{16} \sum_{b+c=d} \eta_{1}(x+b) \eta_{2}(x+b) \eta_{3}(x+b) \eta_{4}(x+b) \bar{\chi}(x+b) \bar{U}(x+b, x+c) \chi(x+c) .
$$

$A_{I}^{\mu}$ and $P_{I}$ satisfy a PCAC relation [37] with the anomaly of four Dirac fermions [23]. Compared to Eq. (3.24) a complication is the need for renormalization [37], but this is analogous to the renormalization of Ward-Takahashi identities of Wilson fermions.

If staggered fermions do indeed provide a $\left(4 n_{f}\right)$-species version of QCD, then general features of the hadron spectrum should provide clear numerical evidence. The chiral symmetries, and the standard line of reasoning for spontaneously broken symmetry, imply that the pseudoscalar meson masses should behave (with degenerate quark masses $m_{q}$ ) as

$$
m_{a \xi}^{2}=2 m_{q} B+a^{2} \Delta_{\xi}+\mu^{2} \delta_{a 0} \delta_{\xi I},
$$

where $a=0, \ldots, n_{f}^{2}-1$ labels flavor and $\xi \in\{P, A, T, V, I\}$ labels taste. The dynamical quantities $B$, $\Delta_{\xi}^{1 / 4}$, and $\mu$ are of order $\Lambda$, the characteristic dynamical scale of the gauge fields. The exact (tastenonsinglet) chiral symmetries of Eq. (3.21) imply $\Delta_{P}=0$. The " $\eta^{\prime}-\pi^{0}$ splitting" parameter $\mu^{2}$ can be generated only for the flavor- and taste-singlet meson. This pattern is shown (for $n_{f}=2$ ) in Fig. 1. It is consistent with extensive numerical simulations in the quenched approximation, as well as with 2 and $2+1$ flavors of (rooted, staggered) sea quarks [10, 39], including the scaling of the pseudoscalar splittings $a^{2} \Delta_{\xi}$ [40]. 
The approach to the continuum limit can be clarified with two theoretical tools, the Symanzik effective field theory $[9,41]$ and chiral perturbation theory $(\chi \mathrm{PT})[41,42,43,44]$. In the Symanzik local effective Lagrangian ( $\mathrm{LE} \mathscr{L}$ ), dimension-four interactions have $\mathrm{SO}(4)$ rotational symmetry and, by design, $\mathrm{SU}_{\mathrm{V}}\left(4 n_{f}\right) \times \mathrm{SU}_{\mathrm{A}}\left(4 n_{f}\right)$ flavor-taste chiral symmetry, softly broken by mass terms. Dimension-six four-quark operators break these down to $\mathrm{SW}_{4}$ and $\Gamma_{4} \times \mathrm{SU}_{\mathrm{V}}\left(n_{f}\right) \times \mathrm{U}_{\mathrm{A}}\left(n_{f}\right)$, as indicated by the embeddings (3.28)-(3.30) [41, 42, 43]. In particular, some of these operators are invariant only under the $\Gamma_{4}$ taste symmetry, not a full SU(4). They are variously called tastebreaking or taste-exchange interactions. The LE $\mathscr{L}$ analysis clarifies why smearing strategies reduce the strength of taste-symmetry breaking [45]. If successful, smeared actions should yield smaller splittings $\Delta_{\xi}$. They do [46]. They also improve the scaling of the $\rho$-meson mass and the static potential [47].

The splittings and other effects of broken taste symmetry are, of course, a complication. They lead to multi-parameter fitting procedures, for example of the pseudoscalar decay constants [48]. Another example is the $\pi$ - $\pi$ threshold, which splits into five $\pi_{\xi}-\pi_{\xi}$ thresholds, $\xi \in\{P, A, T, V, I\}$. In last year's plenary talk on this subject [49], the need for complicating fitting was one reason to deem lattice QCD with rooted staggered quarks "ugly." Many of the complications come not from rooting but from the intricate symmetry structure and the desire for statistically sound fits. Fitting is nicely illustrated by $f_{\pi}$ and $f_{K}$. The staggered data start so close to the experimental result that just about any chiral extrapolation would agree with experiment, but statistically good fits are obtained only when staggered $\chi \mathrm{PT}$ is used [50]. Thus, these fits provide further evidence that staggered (valence) fermions do indeed simulate $4 n_{f}$ flavors of quark.

To study how these properties of staggered fermions relate to gauge-field topology, one must consider the eigenvalues of the staggered Dirac operator. The exact (non-singlet) chiral symmetries imply that eigenvalues come in complex conjugate pairs, $\pm i \lambda_{i}+m_{0}$, with orthonormal eigenvectors $f_{i}(x)$ and $\varepsilon(x) f_{i}(x)$. In a gauge field with a non-zero topological charge $Q$, the continuum Dirac

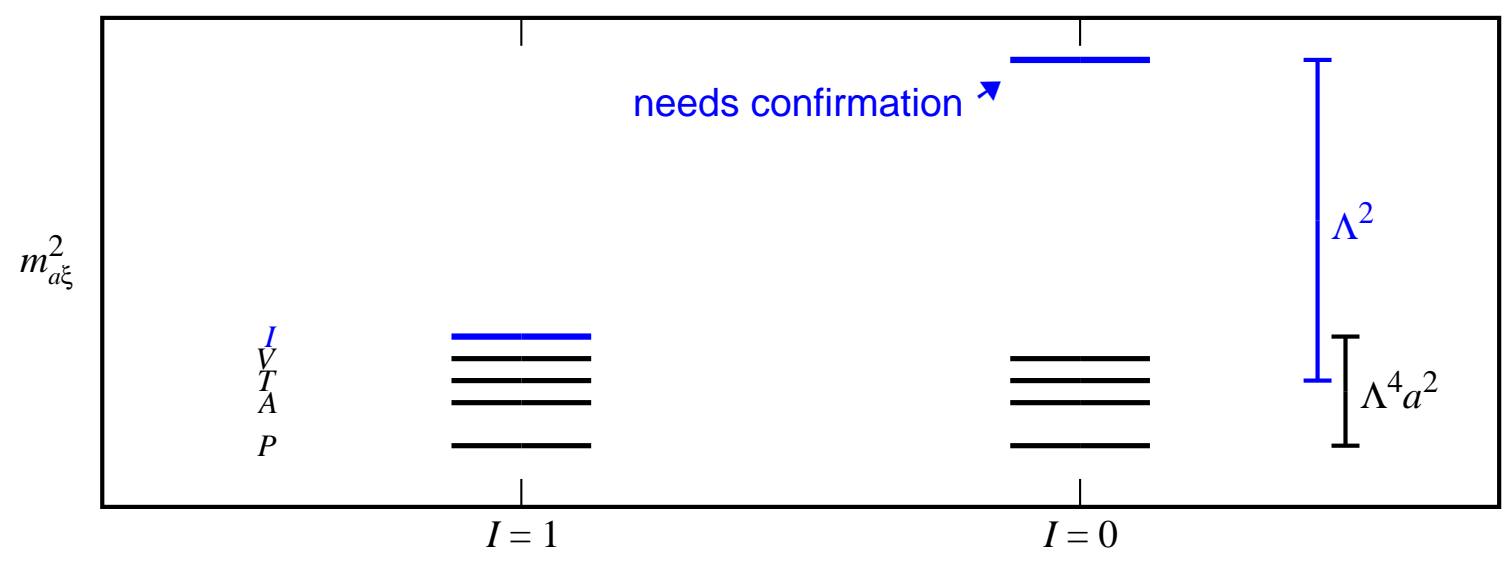

Figure 1: Pseudoscalar spectrum for 2 flavors of staggered fermion, so 8 species in all. The isovector multiplets (with $I_{3}=+1,0,-1$ ) each consist of sixteen states, split by lattice artifacts of order $\Lambda^{4} a^{2}$ into submultiplets with $1,4,6,4$, and 1 states (for, respectively, irreps of taste $I, V, T, A$, and $P$ ). The isosinglet multiplet is similar, except that the taste-singlet $I$ splits from the others by continuum-QCD effects (as usual). This $\eta^{\prime}$-like state suffers from noisy correlators, and numerical data are consistent with this picture without being definitive [39]. 
operator has $n_{ \pm}$zero modes of chirality \pm 1 , satisfying the index theorem $n_{+}-n_{-}=n_{s} Q$, where $n_{s}$ is the total number of species. With lattice staggered fermions, the zero modes are no longer exact. Nevertheless, if staggered fermions do indeed yield four quarks, then several features must emerge dynamically [51]. First, low-lying eigenvalues must cluster in quartets. Second, some (pairs of) eigenvalues should be near-zero modes with $\lambda_{i} \sim \Lambda^{2} a$. Third, the chirality of the corresponding eigenvectors should be close to \pm 1 , in a way that satisfies the index theorem. Finally, in the finitevolume $\varepsilon$ regime, the distributions of eigenvalues should agree with expectations from random matrix theory [52].

Early numerical work was inconclusive [53], but with improved staggered actions, quartets of eigenvalues clearly emerge in numerical simulations [54]. In particular, if the gauge field has topological charge $Q$, then the eigenvalue spectrum contains quartets of the same (taste-singlet, Eq. (3.32)) chirality, such that the index theorem is satisfied [54]. Even with smearing, early comparisons of random matrix theory suggested that staggered fermions are topology blind [55]. Equation (3.33) implies two regimes of interest [56, 57], however,

$$
\begin{aligned}
& \varepsilon \text { regime : } \quad \Lambda \gg L^{-1} \gg m_{\xi}, \forall \xi, \\
& \varepsilon^{\prime} \text { regime : } m_{\xi \neq P} \gg L^{-1} \gg m_{P} .
\end{aligned}
$$

In the $\varepsilon$ regime all "pions" are pseudo-Goldstone bosons, whereas in the $\varepsilon^{\prime}$ regime most of them are very massive particles. In the $\varepsilon$ regime staggered fermions should and do behave like a 4 -species theory [57]; in the $\varepsilon^{\prime}$ regime they should not [56] and do not [55].

In summary, naïve fermions appear to be problematic, because the anomaly is not generated. Analysis of the unexpected doubling symmetries rescues the formulation, however, yielding staggered fermions. Spacetime and flavor-like symmetries become entangled, making the interpretation as Dirac fermions less transparent. But now not only are the exact chiral symmetries non-singlets under a flavor-like quantum number called taste, but also an anomalous taste-singlet axial current can be found. On this basis, the emergence of $4 n_{f}$ Dirac fermions is theoretically plausible. Owing to a wealth of results examining the nonperturbative content and structure, it is also fair to say that the validity of (unrooted) staggered fermions has been established numerically.

\section{Rooting with full SU(4) taste symmetry}

The previous section reviewed some aspects of staggered fermions, focusing on how four species emerge in the continuum limit. Before discussing whether the fourth-root procedure can reduce these four species to one, I would like to present a Gedanken algorithm. The aim is to separate some potentially confusing aspects of rooting, free of the complications of staggered fermions' taste-exchange interactions.

Suppose that an algorithm designer with a good imagination (and a wicked sense of humor) found a way to speed up "your favorite lattice fermions" by substituting

$$
\operatorname{det}_{1}(\not D+m)=\left\{\operatorname{det}_{4}\left[(\not D+m) \otimes \mathbf{1}_{4}\right]\right\}^{1 / 4},
$$

thereby introducing four "tastes." Here $\operatorname{det}_{1}$ is a determinant for $n_{f}$ flavors, with 1 taste per flavor; $\operatorname{det}_{4}$ is for $n_{f}$ flavors, but 4 tastes per flavor. If the determinant is real and positive, this step does not 
change the Monte Carlo weight at all, because the right-hand side is just a clever trick to calculate the left-hand side. Of course, the trick fails if the left-hand side is not real (e.g., for nonzero chemical potential [58]), or if it can be negative (e.g., $m<0$ ).

To probe the dynamics of this system, first introduce sources $\left(J^{a}, J_{5}^{a}\right)$ for scalar and pseudoscalar meson operators $\left(-i \bar{\psi} T^{a} \psi,-i \bar{\psi} T^{a} \gamma_{5} \psi\right)$ in the original theory:

$$
\begin{aligned}
Z\left[J^{a}, J_{5}^{a}\right] & =\int \mathscr{D} \mathscr{U}\left\{\operatorname{det}_{1}\left(\not D+m+J+J_{5} \gamma_{5}\right)\right\}^{N_{r}} \\
& =\int \mathscr{D} \mathscr{U}\left\{\operatorname{det}_{4}\left[\left(\not D+m+J+J_{5} \gamma_{5}\right) \otimes \mathbf{1}_{4}\right]\right\}^{N_{r} / 4},
\end{aligned}
$$

where $\mathscr{D} \mathscr{U}$ is the gauge-field measure (including $e^{-S_{\text {gauge }}}$ ), and the number of "replicas" $N_{r}$ will be useful below. The $T^{a}$ are flavor matrices (with $T^{0}=i 1 / \sqrt{2 n_{f}}$ ), and $J_{(5)}=-i J_{(5)}^{a} T^{a}$.

Spontaneous symmetry breaking is revealed by looking at the Legendre effective action

$$
e^{\Gamma\left(\sigma^{a}, \pi^{a}\right)}=\left.Z\left[J, J_{5}\right] e^{-\sum_{x}\left[J^{a}(x) \sigma^{a}(x)+J_{5}^{a}(x) \pi^{a}(x)\right]}\right|_{J_{(5)}=J_{(5)}(\sigma, \pi)},
$$

where $J_{(5)}(\sigma, \pi)$ are defined implicitly, so that they create prescribed fields $\left(\sigma^{a}, \pi^{a}\right)$ :

$$
\sigma^{a}(x)=\left.\frac{1}{Z} \frac{\partial Z}{\partial J^{a}(x)}\right|_{J_{(5)}=J_{(5)}(\sigma, \pi)}, \quad \pi^{a}(x)=\left.\frac{1}{Z} \frac{\partial Z}{\partial J_{5}^{a}(x)}\right|_{J_{(5)}=J_{(5)}(\sigma, \pi)} .
$$

The mass matrices for $\sigma^{a}$ and $\pi^{a}$ are obtained from second derivatives of $\Gamma$. In the case at hand, they are constrained by symmetry-invariance of the fermion action is expressed in Eqs. (4.2) and (4.3) as the invariance of the determinants under a change of basis. One finds [59]

$$
\begin{aligned}
& \left.\frac{\partial^{2} \Gamma}{\partial \sigma^{a} \partial \sigma^{c}} f^{c d b} \sigma^{d}\right|_{\sigma_{0}, \pi_{0}}+\left.\frac{\partial^{2} \Gamma}{\partial \sigma^{a} \partial \pi^{c}} f^{c d b} \pi^{d}\right|_{\sigma_{0}, \pi_{0}}=0, \\
& \left.\frac{\partial^{2} \Gamma}{\partial \pi^{a} \partial \pi^{c}} d^{c d b} \sigma^{d}\right|_{\sigma_{0}, \pi_{0}}+\left.\frac{\partial^{2} \Gamma}{\partial \pi^{a} \partial \sigma^{c}} d^{c d b} \pi^{d}\right|_{\sigma_{0}, \pi_{0}} \propto \text { mass and anomaly terms, }
\end{aligned}
$$

where the vacuum fields $\left(\sigma_{0}, \pi_{0}\right)$ are those in the absence of sources $J_{(5)} \cdot{ }^{4}$ If one assumes that all vacuum fields vanish except the flavor-singlet scalar $\sigma_{0}^{0}$, then one obtains a constraint

$$
\left.\frac{\partial^{2} \Gamma}{\partial \pi^{a} \partial \pi^{b}}\right|_{\sigma_{0}, \pi_{0}} \propto \text { mass and anomaly terms, }
$$

and no constraint on $\partial^{2} \Gamma / \partial \sigma^{a} \partial \sigma^{b}$. Equation (4.8) is the basis for formulae such as Eq. (3.33). Of course, the dynamical assumption that $\sigma_{0}^{0} \neq 0$ has not been proven mathematically but has been established numerically with various types of lattice fermions.

Equation (4.3) apparently has more symmetry than Eq. (4.2)—SU $\left(4 n_{f}\right) \times \mathrm{SU}\left(4 n_{f}\right)$ instead of $\mathrm{SU}\left(n_{f}\right) \times \mathrm{SU}\left(n_{f}\right)$. I will call the extended symmetry a phantom symmetry, because it is a figment of an algorithm designer's imagination. One can study the dynamical consequences of the phantom symmetry by promoting the sources to taste-nonsinglets [17]:

$$
Z\left[J^{A}, J_{5}^{A}\right]=\int \mathscr{D} \mathscr{U}\left\{\operatorname{det}_{4}\left[(\not D+m) \otimes \mathbf{1}_{4}+J+J_{5} \gamma_{5}\right]\right\}^{N_{r} / 4},
$$

\footnotetext{
${ }^{4}$ Instead of fixing $J_{(5)}$ in Eq. (4.5) to get prescribed fields, simply set $J_{(5)}=0$ on the right-hand side to get $\left(\sigma_{0}, \pi_{0}\right)$.
} 
where the flavor-taste generator index $A$ now runs from 0 to $\left(4 n_{f}\right)^{2}-1$. One finds the same pattern of spontaneous symmetry breaking as in Eqs. (4.6)-(4.7), leading to mass relations like (4.8) also for the taste nonsinglet phantom pseudoscalars. In all, there are $\left(4 n_{f}\right)^{2}-1$ pseudo-Goldstone bosons, instead of just $n_{f}^{2}-1$, and one taste- and flavor-singlet $\eta^{\prime}$-like meson.

But what should one make of the phantoms? The equivalence of the two formulations, (4.2) and (4.3), shows that phantoms do not exist. The symmetry analysis suggests they do. (A numerical simulation of your favorite rooted fermions could look at taste-nonsinglet correlators to decide and would probably find them.) The conundrum is easily resolved if the phantoms violate unitarity and cancel each other out in correlators that are oblivious to taste [17]. The lack of unitarity, particularly when constrained by symmetry, is not a concern and, in fact, is familiar in gauge theories [60].

The Gedanken algorithm helps establish a foundation for some questions concerning rooted staggered fermions. First, we see what kinds of correlators are physical. For bosonic taste, these are taste singlets and anything related to taste singlets by symmetry. ${ }^{5}$ For fermionic taste, one needs single-taste correlators [49]. It is possible to construct unphysical correlators, which could lead to confusion or mistakes. Another use of Eqs. (4.2) and (4.3) is to set a criterion for proving staggered fermions incorrect. Any argument that would also kill the Gedanken algorithm simply does not pertain to the issues at hand. An example is the proof that $(\not D+m)^{1 / 4}$ is non-local [61], which does not prove that $\left[\operatorname{det}_{4}(\not D+m)\right]^{1 / 4}$ cannot be obtained from a path integral with a local action [62]. Attempts to disprove staggered fermions must focus on features not shared with the Gedanken algorithm, namely the violations of perfect SU(4) taste symmetry. Finally, note that the details of the phantom sector depend on $N_{r}$. For $N_{r} \in \mathbb{Z}$, the rooted formulation of Eq. (4.3) is identical to a manifestly sound quantum field theory. On the other hand, Eqs. (4.2) and (4.3) show that irrational choices, like $N_{r}=\pi$, are simply irrational.

\section{Rooting with staggered fermions}

We are now ready to discuss rooted staggered fermions, which is the Ansatz that quarks can be simulated with Eq. (1.1), using any of several available algorithms [63, 20]. An initial set of arguments in favor are as follows. First, Eq. (1.1) resembles Eq. (4.9), especially when one looks at the $\not_{\text {stag }}$ defined via Eq. (3.27); the four-taste determinant has SU(4) taste-violating parts that are suppressed superficially by powers of the lattice spacing. Second, perturbative renormalization [26, 38] and the nonperturbative features discussed at the end of sect. 3 support the picture of unrooted staggered fermions as a QCD-like theory, in which violations of SU(4) taste symmetry vanish in the continuum limit. Finally, having thus achieved a $4 \times 4$ block structure, the fourth root is as straightforward as in sect. 4.

Implicit in this line of reasoning is the assumption that the continuum limit and rooting commute. We shall come back to this below. First, however, I would like to consider objections that hold even if these arguments are all correct. In particular, I stipulate for now that $\mathrm{SU}\left(4 n_{f}\right)$ flavortaste symmetry emerges in the continuum limit, citing the evidence presented at the end of sect. 3.

In the context of rooting most of the $\mathrm{SU}\left(4 n_{f}\right)$ symmetry is a phantom symmetry. As such one should ask whether a phantom sector of particles is seen in numerical simulation. (They are, in the scalar propagator, see below.) If so, they violate unitarity. Moreover, at non-zero lattice spacing the

\footnotetext{
${ }^{5}$ For example, one may use the taste- $P$ irrep for pseudoscalar mesons, the taste- $V$ irrep for vector mesons, etc.
} 
symmetry is smaller than $\mathrm{SU}\left(4 n_{f}\right)$, as exhibited in (3.29) and (3.30). Consequently, the phantoms are not degenerate, so they will not cancel identically in physical correlators. Fortunately, sect. 4 shows in general (if sketchy) terms that the rooted dynamics provide a safe house for phantoms, when (as stipulated above) they become degenerate in the continuum limit. Then the phantom sector is benign, even if it is not unitary, because the cancellation becomes perfect.

The ramifications of non-degenerate phantoms are still ugly, in some cases perhaps even scary. A useful and illuminating tool is $\chi \mathrm{PT}$ adapted for the case at hand: rooted, staggered chiral perturbation theory (RS $\chi \mathrm{PT}$ ) [64]. This extension of partially quenched $\chi \mathrm{PT}$ encodes the $N_{r}$ dependence, so it is sensitive to the dynamics of the sea. As mentioned in sect. 3 , the fit to pseudoscalar meson masses and decay constants provides clear evidence that this description works. To isolate the sensitivity of the numerical data to the sea quarks, MILC now tries fits in which the number of replicas floats. At this conference Bernard reported [65] (in his notation, $N_{r}=4 n_{r}$ )

$$
\frac{1}{4} N_{r}=n_{r}=0.28(2)(3),
$$

in striking agreement with the expectation $1 / 4$. Further evidence comes from heavy-light decay constants. Continuum partially quenched $\chi$ PT blatantly fails to describe the numerical data [66], while RS $\chi$ PT yields a statistically sound fit [4]. The topological susceptibility is also betterdescribed with $\mathrm{RS} \chi \mathrm{PT}$ than with continuum $\chi \mathrm{PT}[67]$.

The pseudoscalar mesons just mentioned are low-lying states, but higher mass particles must be considered too. Correlators, for example those of glueballs, contain not only the single hadron of interest but also two-phantom-particle states. It can become hard to determine the single-particle mass, but these correlators are still interesting, because the two-particle contribution probes the sea [68]. Correlators of taste-singlet scalar mesons contain $\pi_{\xi}-\eta_{\xi}$ pairs, $\xi=P, A, T, V$, and $\pi_{\xi}$ and $\eta_{\xi}$ consist of a valence and a sea quark. These $\pi_{\xi}-\eta_{\xi}$ bubbles can be described with $\chi$ PT, analogously to the quenched scalar correlator [69]. The bubbles' weights depend on $N_{r}$. For $N_{r}<4$ some weights are negative, as one expects when unitarity is violated. Once all thresholds become degenerate, the bubbles always add up to the correct total. Comparisons of numerical data with the $\mathrm{RS} \chi \mathrm{PT}$ formulae confirm this picture [70], supporting the correctness of the rooted sea. A similar story holds in the case of a mixed action with a rooted sea and Ginsparg-Wilson valence quarks; the numerical data are again well described by mixed-action $\chi$ PT [71].

In summary, the violations of unitarity are a serious objection and can be a practical obstacle. The numerical data suggest, however, that these effects, including their influence on the error budget, can be managed with RS $\chi$ PT. Furthermore, the analysis of sect. 4 reveals a safe house for phantoms, not only for pseudoscalar mesons, but also for other hadrons.

A distinct, but related, issue is the locality of rooted staggered fermions. Critics of the rooting procedure have long suspected non-local behavior [72], but to my knowledge the concern is that a Lagrangian of the form $\bar{\psi}(\not D+m)^{1 / 4} \psi$ is non-local [61]. Because this line of attack would kill the manifestly correct Gedanken algorithm, this concern is a red herring. There is, however, another kind of non-local behavior. Suppose one writes

$$
\operatorname{det}_{4}\left(\not \not_{\text {stag }}+m\right)=\left[\operatorname{det}_{1}\left(\not_{\mathrm{SU}(4)}+m\right)\right]^{4} T
$$

where the first factor on the right-hand side is manifestly SU(4) symmetric. The remaining factor $T$ cannot represent a set of local interactions for the gauge field, because the left-hand side generates 
a spectrum with taste splittings, and the SU(4)-symmetric determinant does not [73]. It is certainly accurate to call this a non-locality, but I am not sure how illuminating it is. Before taking the fourth root, the non-locality is particle-like. After taking the fourth root on both sides of Eq. (5.2), it strikes me as plausible that the non-locality of $T^{1 / 4}$ does not ruin cluster decomposition, it may become local in the continuum limit [74], and it certainly is not the non-locality of a propagator $(\not D+m)^{-1 / 4}$. Thus, it is not the non-locality that critics seem to have in mind.

To be confident that the continuum limit is correct, it would be helpful if one could establish a Symanzik LE $\mathscr{L}$, not with an Ansatz based on symmetries, but through a derivation from the underlying lattice field theory. The Symanzik structure is evident at the tree level, and it seems to be accepted without controversy at all orders in perturbation theory. (It is not, however, proven at the level needed to prove Reisz's theorems [75]. An important first step has been to extend the power-counting theorem to staggered fermions [76].) In a forthcoming paper, Bernard, Golterman, and Shamir (BGS) show a new way to do so. For a short version with more details than I can give here, see Golterman's talk at this conference [77].

The new work of BGS is based on Shamir's block-spin renormalization group [78], which was covered thoroughly in Sharpe's review [49] last year. After $n$ blocking steps, Shamir arrives at a blocked staggered operator

$$
(\not D+m)_{n} \otimes \mathbf{1}_{4}+a \Delta_{n}
$$

with a SU(4)-symmetric part $(\not D+m)_{n} \otimes \mathbf{1}_{4}$ and a taste-breaking defect $\Delta_{n}$. The blocked quantities live on a lattice with spacing $2^{n} a$, and in the continuum limit $n \rightarrow \infty, a \rightarrow 0, a_{c}=2^{n} a$ fixed. A power-law divergence in $\Delta_{n}$ is expected to be $a_{c}^{-2}$, implying that the combination $a \Delta_{n}$ vanishes in the continuum limit.

The blocked determinant resembles Eq. (4.9), with sources set to provide $a \Delta_{n}$, and $\mathscr{D} \mathscr{U}$ the complicated measure for the gauge field in Ref. [78]. BGS develop an expansion in the underlying spacing $a$ with the aid of some bookkeeping devices. The determinant is generalized to be

$$
\left\{\operatorname{det}_{4}\left[(\not D+m)_{n} \otimes \mathbf{1}_{4}+a \Delta_{n}\right]\right\}^{n_{r}} \rightarrow\left\{\operatorname{det}_{1}\left[(\not D+m)_{n}\right]\right\}^{N_{s}} \frac{\left\{\operatorname{det}_{4}\left[(\not D+m)_{n} \otimes \mathbf{1}_{4}+t a \Delta_{n}\right]\right\}^{n_{r}}}{\left\{\operatorname{det}_{4}\left[(\not D+m)_{n} \otimes \mathbf{1}_{4}\right]\right\}^{n_{r}}}
$$

where $N_{s}$ need not equal $N_{r}=4 n_{r}$, and $t$ need not equal 1 . Sources for valence fermions are

$$
\exp \left\{\bar{\eta}\left[(\not D+m)_{n} \otimes \mathbf{1}_{4}+v a \Delta_{n}\right]^{-1} \eta\right\}
$$

where, again, for bookkeeping $v$ need not equal 1. Post-analysis, one may set $N_{s}=N_{r}, t=v=1$.

Expressions (5.4) and (5.5) can be expanded in $t$ and $v$, justified by the small quantity $a \Delta_{n}$. The double-expansion can then be reverse-engineered to reproduce the Lee-Sharpe LE $\mathscr{L}$ for staggered fermions [41]. Each bookkeeping parameter brings an advantage. Possible nonperturbative nonpolynomial dependence of the LE $\mathscr{L}$ on $N_{s}$ is kept, because other factors are expanded in $t$ and $v$. The expansion in $t$ ensures that, to any order in $a$, the dependence on $n_{r}$ is polynomial, so analytical continuation from an integer to $1 / 4$ is allowed. The expansion in $v$ shows that valence quarks control the symmetries and the field content of the LE $\mathscr{L}$, which do not depend on $n_{r}$. Pending confirmation of the assumptions built into the blocking [78, 49], these are a strong results.

This analysis underscores the importance of checking numerically that $\Delta_{n}$ scales in such a way as to justify the expansions. There are several pieces of evidence to suggest it does. Exhibit A is all experience with anomalous dimensions in QCD. Exhibit B is all experience with the pseu- 
doscalar spectrum as taste-breaking of valence fermions is reduced $[46,10]$; heuristically this is like reducing $v$ without reducing $a$. Lastly, Exhibit C is a pilot investigation of $\Delta_{n}$ itself [79].

A lack of scaling of $a \Delta_{n}$ would kill rooted staggered fermions, because then we would be left with a non-unitary, non-local theory, even in the continuum limit. It is therefore worth stating how the evidence given above could be misleading. The pilot investigation [79] is not yet definitive. The pseudoscalar splittings probe only four of the plethora of taste-exchange effects generated by $\Delta_{n}$. Finally, it could be that rooting and the continuum limit do not commute, in a way so profound that the rooted determinant itself generates $\Delta_{n}$ 's anomalously large anomalous dimension [49]. Such "self-inconsistency" is possible, but highly implausible.

\section{Explicit refutation of Refs. $[12,13,14,15,16]$}

With the preceding sections' outline of rooted staggered quarks as a basis for discussion, I can now address Mike Creutz's specific criticisms, which below are summarized in slanted font. Many of my counterarguments are the same as in the original refutation [17].

\subsection{Order of limits}

Rooted staggered fermions require a "peculiar" order of limits, $a \rightarrow 0$ with $m$ fixed, followed by $m \rightarrow 0$ [12, 13, 14]. The required order is especially peculiar with one flavor [13].

For two or more flavors, this criticism is misguided. Computers have a finite memory, so one takes the continuum limit, $a \rightarrow 0$, at fixed spatial volume, $L^{3}$. But there is no spontaneous symmetry breaking in a finite volume. To select a finite-volume vacuum close to the infinitevolume spontaneously-broken vacuum, explicit symmetry breaking is needed. For this general reason, one should keep $m \neq 0$ while taking the continuum limit, then take $L \rightarrow \infty$, and last $m \rightarrow 0$.

Also, the continuum limit must be carefully specified whenever exact symmetries imply pseudoGoldstone bosons [80]. Suppose there are two kinds of particles with masses $m_{\pi}^{2} a^{2}=\kappa \Sigma, m_{\sigma}^{2} a^{2}=$ $\Sigma^{2}$, and consider an unconventional family of continuum limits, $\kappa=m_{q} a^{1+p}, \Sigma=\Lambda a^{1-p}$. Then $m_{\pi}^{2}=m_{q} \Lambda, m_{\sigma}^{2}=\Lambda^{2} a^{-2 p}$, so if $p>0$ then $\pi$ 's correlation length diverges while $\sigma$ 's does not. This is a different universality class than the standard one, $p=0$. Staggered fermions (without rooting) are subtler still, because the would-be $\pi$-like particles, except the one with taste $P$, have a mass $m_{\xi}^{2} a^{2}=\kappa \Sigma+\Sigma^{4}, m_{\xi}^{2}=m_{q} \Lambda+\Lambda^{4} a^{2-4 p}$. For $p>1 / 2$, the continuum limit strands these at the cutoff, reminiscent of the $\varepsilon^{\prime}$ regime.

With one flavor there is no spontaneous symmetry breaking - the lowest-lying pseudoscalar is an $\eta^{\prime}$-like meson. In this case, the above considerations no longer apply. But every numerical lattice QCD calculation has unphysically large quark masses, to allow the algorithms to run faster, so I do not consider the order of limits to be a serious criticism even with one flavor.

\subsection{Mutilated quark-mass dependence}

Rooted staggered fermions yield the same system for $-m$ as for $m$; therefore, the small-mass behavior is a function of $\mathrm{m}^{2}$, which we know is wrong in specific cases [12, 13, 14].

Odd powers of the quark mass stem from zero modes of $\not D$. As is obvious from Eq. (4.1), the rooting procedure turns $m$ into $\left(m^{4}\right)^{1 / 4}=|m|$. This defect of the algorithm has nothing to do with staggered fermions [17]. 
It is instructive to examine how odd powers arise when one has near-zero modes $\lambda_{i} \sim \Lambda^{2} a$, as with staggered fermions. Two pairs with eigenvalue $\pm i c_{i} \Lambda^{2} a, i=1,2$ arise [54], so

$$
\left[\operatorname{det}_{4}\left(\not D_{\text {stag }}+m\right)\right]^{1 / 4} \propto\left[\left(c_{1}^{2} \Lambda^{4} a^{2}+m^{2}\right)\left(c_{2}^{2} \Lambda^{4} a^{2}+m^{2}\right)\right]^{1 / 4} \approx|m|\left[1+\frac{1}{4}\left(c_{1}^{2}+c_{2}^{2}\right) \frac{\Lambda^{4} a^{2}}{m^{2}}\right]
$$

when the limits are taken in the correct order. If one takes the limits in the wrong order, then the determinant is indeed a function of $m^{2}$. It is correct to consider this the wrong theory, but it is incorrect to assert that the numerical work takes the limit in the wrong order. The applicability of Eq. (6.1) has been demonstrated clearly in the Schwinger model [81]. It is also possible to follow the $m$ dependence of observables down until $m \sim \Lambda^{3} a^{2} \ll \Lambda^{2} a$ using RS $\chi$ PT [82], well beyond the regime where the approximation in Eq. (6.1) applies.

\subsection{Cancellation of non-unitary contributions}

The cancellations among taste multiplets seem contrived [12, 13, 14].

The Gedanken algorithm of sect. 4 shows that phantoms cancel each other automatically in the absence of taste-exchange processes [17]. Thus, if the Shamir defect scales so that one can treat it as a perturbation, it is not reasonable to call the cancellation contrived. The scalar propagator provides a good numerical test, for two reasons. First, the weights of the non-unitary contributions depend (in $\chi \mathrm{PT}$ ) on $N_{r}$ in an illuminating way, which could be checked numerically with (some) new simulations with $N_{r}=1,2,3,4$. Second, the cancellation of the phantom modes depends on $a^{2}$, which is being monitored as part of ongoing simulations [70].

\subsection{Rank of chiral symmetry}

The rank (i.e., number of diagonal generators) of the chiral symmetry is wrong [13, 14].

With four tastes the rank is indeed larger. But the Gedanken algorithm shows that this is not a feature of staggered fermions per se, but of the rooting procedure itself. It provides a clear explanation of why the physical sector is the taste-singlet sector. The other particles are phantoms. In particular the extra neutral pseudoscalars cancel each other out, perfectly in the Gedanken algorithm and (on the basis of numerical results) to order $a^{2}$ with rooted staggered fermions.

\subsection{Anomalies}

The conventional axial anomaly cancels $[12,13,14]$.

This claim is simply wrong. The taste- $P$ axial current suffers no anomaly, as desired, because it is off-diagonal in taste. The conventional staggered anomaly appears in the taste-singlet PCAC relation, as explained in the discussion of Eqs. (3.31) and (3.32). It comes with the right strength, because rooting multiplies the 4 -species anomaly with the factor $\frac{1}{4}$ appropriate for one species.

\subsection{Topology}

Rooted staggered quarks implement topology incorrectly, because quartets must break up at the boundary of topological sectors $[12,14,15]$. Rooting averages over positive and negative chirality modes, so the index theorem cannot be satisfied [13].

Studies of the eigenvalues reveal a quartet structure, including quartets of near-zero modes [54]. As the gauge field transits from one topological sector to another, this structure must indeed be dis- 
rupted. But gauge fields near the boundary of topological sectors should have a large gluon action and are, hence, suppressed. If not, it is a drawback of the gluon action and has nothing to do with staggered fermions. It would be interesting to monitor this with smooth gauge fields. It would bode well for rooting if the quartets rearrange themselves rapidly, similarly to the way a lattice approximant to $Q$ does.

The assertion about positive and negative chirality is false. Ref. [57] shows that near-zero modes appear in quartets of the same taste-singlet chirality, Eq. (3.32). The confusion may stem, as in sect. 6.5, from contemplating taste- $P$ currents and densities. But taste- $P$ chirality vanishes for all modes, including near-zero modes: $\sum_{x} f_{i}^{\dagger}(x) \mathcal{\varepsilon}(x) f_{i}(x)=0$, because if $f_{i}(x)$ is an eigenvector of eigenvalue $i \lambda_{i}+m$, then $\varepsilon(x) f_{i}(x)$ is the eigenvector of $-i \lambda_{i}+m$, and they are orthogonal. Hence, many statements about chirality in Refs. $[12,13,14,15,16]$ are simply ill-conceived.

\section{7 't Hooft vertices}

't Hooft vertices generate contributions to correlation functions that diverge in the chiral limit as a power of $m^{-1}[15,16]$.

Only a full analysis of 't Hooft vertices can refute this assertion, because no explicit equations are given in Refs. $[15,16]$. Here I shall examine the $\eta^{\prime}$-like meson with one quark flavor, which should $[15,16]$ expose the problem. The taste-singlet propagator consists of two terms

$$
\begin{aligned}
C(x, y) & =\operatorname{tr}\left[G(x, y) U_{5} G(y, x) U_{5}\right], \\
D(x, y) & =\operatorname{tr}\left[G(x, x) U_{5}\right] \operatorname{tr}\left[G(y, y) U_{5}\right],
\end{aligned}
$$

where the trace is over color, and $U_{5}$ abbreviates the sign factors, link matrices, and translations indicated in Eq. (3.32). The quark propagator $G(x, y)=\langle\chi(x) \bar{\chi}(y)\rangle_{\chi, \bar{\chi}}$. The right way to combine $C(x, y)$ and $D(x, y)$ is

$$
\left\langle\eta^{\prime}(x) \eta^{\prime}(y)\right\rangle=\left\langle-\frac{1}{4} C(x, y)+\frac{1}{16} D(x, y)\right\rangle_{U},
$$

where the sign arises from Fermi statistics. The weights $\frac{1}{4}$ and $\frac{1}{16}$ are crucial and follow immediately from Eqs. (4.2) and (4.3).

't Hooft vertices arise from (near) zero modes. At first glance, such gauge fields are suppressed by small eigenvalues from the determinant. For rooted staggered fermions the determinant factor is that given in Eq. (6.1). In a correlation function, however, these factors can be cancelled by small eigenvalues in the denominator, coming from fermion propagators.

Let us consider the $Q=1$ sector for simplicity. Numerical simulation [54] tells us there are four near-zero modes, which we shall label $\pm 1, \pm 2$. Inserting an eigenvector-eigenvalue representation of the fermion propagators, the disconnected contribution is

$$
D(x, y)=\sum_{i= \pm 1, \pm 2} \frac{1}{i \lambda_{i}+m} f_{i}^{\dagger}(x) U_{5} f_{i}(x) \sum_{j= \pm 1, \pm 2} \frac{1}{i \lambda_{j}+m} f_{j}^{\dagger}(y) U_{5} f_{j}(y)+\ldots \sim\left(\frac{4}{m}\right)^{2},
$$

isolating the most singular parts as $m \rightarrow 0$ (with $\lambda \ll m$ ). Similarly, the connected correlator is

$$
C(x, y)=\sum_{i, j} \frac{1}{i \lambda_{i}+m} f_{i}^{\dagger}(x) U_{5} f_{j}(x) \frac{1}{i \lambda_{j}+m} f_{j}^{\dagger}(y) U_{5} f_{i}(y) .
$$

Within a quartet, it is plausible to assume $f_{i}^{\dagger}(x) U_{5} f_{j}(x) \sim \mathrm{O}(a), i \neq j$, because each eigenvector should have a different taste. Then, 


$$
C(x, y)=\sum_{i= \pm 1, \pm 2}\left(\frac{1}{i \lambda_{i}+m}\right)^{2} f_{i}^{\dagger}(x) U_{5} f_{i}(x) f_{i}^{\dagger}(y) U_{5} f_{i}(y)+\ldots \sim \frac{4}{m^{2}} .
$$

When combining the two pieces according to Eq. (6.4), no singular behavior appears as $m \rightarrow 0$ (after $a \rightarrow 0$ ). Thus, modulo one easy-to-check assumption, Creutz's claims about the 't Hooft vertex do not hold up in this, the simplest, example. Similarly, the 't Hooft vertex of the physical eightfermion operator discussed in Ref. [16] is not singular, when Fermi statistics and orthogonality in taste is taken into account [83].

\subsection{Summary}

The criticisms of Refs. [12, 13, 14, 15, 16] are based on three sources of confusion. Difficulties with mass dependence, the rank of the flavor-taste symmetry, and the way that phantoms cancel are explained by the Gedanken algorithm of sect. 4. This approach also illuminates the blunder of confusing the exact taste-nonsinglet chirality with the conventional taste-singlet chirality, which connects correctly to topology and 't Hooft vertices. Finally, it is easy to draw incorrect conclusions by choosing the incorrect order of limits (obdurately forcing $m \rightarrow 0$ before $a \rightarrow 0$ ). The correct order is not "absurd" $[12,14]$, but necessary whether or not staggered fermions are employed.

\section{New developments}

There are two noteworthy methodological developments in improved actions for staggered fermions. One is a new discretization reducing taste-exchange interactions, while maintaining $\mathrm{O}\left(a^{2}\right)$ improvement. The other is the completion of the full $\mathrm{O}\left(\alpha_{s} a^{2}\right)$ corrections to the gluon action. Either or both could be incorporated into future simulations of sea quarks.

\subsection{Highly improved staggered quarks}

Although the Asqtad action has much smaller taste-changing effects than the original staggered action, it would be better to reduce them further. The Asqtad action is obtained from the standard staggered action, Eq. (3.8), in two steps, as follows. First smeared links are constructed,

$$
V_{\mu}=\mathscr{F}_{\mu} U_{\mu}=\prod_{\rho \neq \mu}^{\text {sym }}\left(1+\frac{a^{2}}{4} \triangle_{\rho}\right) U_{\mu},
$$

where $\triangle_{\rho}$ is a covariant nearest-neighbor second derivative, and the product is symmetrized over all possible orderings of the directions orthogonal to $\mu$. It yields bent staples of length 3,5 , and 7; substituting $V$ for $U$ in Eq. (3.8) yields the FAT7 action. The smearing is designed for, and is successful at, reducing the size of taste-exchange interactions. It does not achieve Symanzik improvement, however. This is achieved by adding two improvement terms to the FAT7 action, the Naik [84] term and the Lepage term [9], to obtain the $a$-squared (Asq) action and, with tadpoleimproved couplings, the Asqtad action.

A new action [85] extends the philosophy behind the FAT7 and Asqtad actions, introducing $W_{\mu}=\mathscr{F}_{\mu} \mathscr{U} \mathscr{F}_{\mu} U_{\mu}$, where the operator $\mathscr{U}$ brings the smeared $V$-links back into $\mathrm{U}(3)$, thereby reducing ultraviolet fluctuations, including taste-changing interactions [86]. Using $W$ in Eq. (3.8) and generalizing the Naik and Lepage terms yields the highly-improved staggered quark (HISQ) action [85]. A numerical implementation [87] is only around two times slower than Asqtad. 
The first results from the HISQ action shed light on the issues of sect. 5. Reference [85] calculates in perturbation theory the size of the four-quark interactions in the Symanzik LE $\mathscr{L}$ for both Asqtad and HISQ, finding the latter's to be an order of magnitude smaller. Reference [85] also computes the $a^{2} \Delta_{\xi}$ splittings of Eq. (3.33), finding them to be half as large with HISQ. In the language of (5.4) and (5.5), the HISQ action reduces the size of the (valence) Shamir defect, $v a \Delta_{n}$, by methodological means, and yields a valence spectrum closer to that of the continuum limit.

\section{2 $n_{f}$ dependence of the gauge action}

The MILC ensembles [10] use the order- $a^{2}$ improved gauge action [88]. The pure-gauge one-loop matching of this action has been available for a long time [89]. Using these improved couplings, as MILC does, removes errors formally of order $\alpha_{s} N_{c} a^{2}$ but not $\alpha_{s} n_{f} a^{2}$. Earlier this year, Hao et al. completed the fermion loop calculation with Asqtad fermions [90]. They find that the fermion loop has the opposite sign from the gluon+ghost loops. For $n_{f}=3$ they change the sign of the radiative correction to the coupling of the rectangle and reduce greatly the radiative correction to the twisted parallelogram. They recommend putting these results into future simulations and note that the size of the effects is what is needed to explain observed scaling violations [91]. The MILC Collaboration plans to use this result in future simulations [92].

\section{Conclusions}

Staggered fermions are fast, but not easy. After trivializing the spinor index of the naïve fermion field, the projection to one component entangles flavor symmetries with spacetime symmetries, and the remaining species doubling is reflected in a new flavor-like quantum number, taste. The exact flavor and taste symmetries are expected to enlarge to (softly broken) $\mathrm{SU}_{\mathrm{V}}\left(4 n_{f}\right) \times$ $\mathrm{SU}_{\mathrm{A}}\left(4 n_{f}\right)$, a mechanism that has been established by numerical simulations.

When the fourth root is taken, staggered fermions have both too little symmetry (discretization effects break $\mathrm{SU}_{\mathrm{V}}\left(4 n_{f}\right) \times \mathrm{SU}_{\mathrm{A}}\left(4 n_{f}\right)$ ) and too much (the target symmetry is $\mathrm{SU}_{\mathrm{V}}\left(n_{f}\right) \times \mathrm{SU}_{\mathrm{A}}\left(n_{f}\right)$ ). Confusion stemming from too much symmetry can be avoided via the Gedanken algorithm discussed in sect. 4. It explains why rooted theories have "extra" particles, in particular pseudoGoldstone bosons, and why the extra symmetry protects physical (single-taste) correlators. Most of the criticisms of Refs. [12, 13, 14, 15, 16] can be refuted with this framework [17, 83].

Valid criticisms of rooted staggered fermions should focus on the difference between staggered fermions and the continuum, namely on the interactions that break the full $\mathrm{SU}_{\mathrm{V}}\left(4 n_{f}\right) \times \mathrm{SU}_{\mathrm{A}}\left(4 n_{f}\right)$ taste-flavor symmetry. The most glaring issue is the violation of unitarity. With the full symmetry such violations cancel identically (for physical correlators). Without the full symmetry they do not cancel, but rooted staggered chiral perturbation theory ( $\mathrm{RS} \chi \mathrm{PT})$ offers a way to describe them. Fits to pseudoscalar masses and decay constants (light and heavy-light), and studies of the two-particle contribution to the scalar propagator, give evidence that $\mathrm{RS} \chi \mathrm{PT}$ works. It not only guides the chiral extrapolation but also provides a framework for estimating the associated uncertainty.

Refutation of specific criticisms, here and in Refs. [17, 83], does not prove that rooted, staggered fermions are valid. It is remarkable, however, that so many numerical tests have shored up the theoretical framework, when any one of them could have gone wrong. Of special interest here is the interplay of chirality and topology. The analysis of chirality in Refs. $[12,13,14,15,16]$ is 
wrong, because it discusses a taste-nonsinglet chiral symmetry that has nothing to do with topology. The conventional staggered taste-singlet chirality agrees with the index theorem, in the same way any two lattice definitions of topological charge do.

More tests can and should be carried out. A comprehensive study of the scaling of the Shamir defect, $a \Delta_{n}$, should help decide whether all taste breaking interactions vanish in the continuum limit. For self-consistency, this study should be done with a rooted staggered sea. Tests of the sea, such as the scaling of the two-particle contributions to taste-singlet correlators, would bolster confidence in RS $\chi$ PT. Most of Creutz's discussion of 't Hooft vertices can be refuted with the setup in sect. 4 , but it does bring out the need to check numerically whether the members of near-zero mode quartets are all of different taste.

Much of our structural, and not to mention practical, understanding of rooted, staggered fermions depends on RS $\chi$ PT. It is based on plausible arguments [64], with one reservation. An original justification for $\chi \mathrm{PT}$ is that it gives the most general description of particle interactions, consistent with unitarity [93]. What happens when unitarity is lost? Can a non-unitary $\chi$ PT describe a non-unitary gauge theory? Is, for example, cluster decomposition enough [94]? This is a basic concern wherever partially quenched $\chi \mathrm{PT}$ is used. With other formulations of lattice fermions one could, if necessary, avoid it. With staggered fermions it seems essential.

A friend of mine, who expects that Eq. (1.1) is not valid, says that the burden of proof is on the staggered community. He is correct, of course, but only half correct. A "proof" is unlikely to be mathematically rigorous. Instead, as in all of lattice gauge theory, methods will be validated, or not, with a combination of theoretical framework and numerical simulation. It does not make much sense for proponents to prove to themselves that their methods are acceptable. Skeptics need to be engaged, examine the theoretical and numerical evidence in favor of rooted staggered fermions, think about the issues clearly, and state where the shortcomings lie. In other words, their job now is to be like Mike. Think it through and write it up!

\section{Acknowledgments}

I would like to thank Mike Creutz for agreeing to the format of our talks and, most of all, for documenting his criticisms in the literature, where they can be scrutinized openly. While preparing the oral and written versions of this talk, I benefited from conversations and e-mail correspondence with David Adams, Christopher Aubin, Jon Bailey, Bill Bardeen, Claude Bernard, Poul Damgaard, Christine Davies, Carleton DeTar, Stefan Dür, Eduardo Follana, Elizabeth Freeland, Maarten Golterman, Alistair Hart, Peter Hasenfratz, Georg von Hippel, Jack Laiho, Yigal Shamir, Steve Sharpe, Doug Toussaint, and Ruth Van de Water.

\section{References}

[1] C.T.H. Davies et al. [HPQCD, MILC, and Fermilab Lattice Collaborations], High-precision lattice QCD confronts experiment, Phys. Rev. Lett. 92 (2004) 022001 [hep-lat/0304004].

[2] C. Aubin et al. [Fermilab Lattice, MILC, and HPQCD Collaborations], Semileptonic decays of D mesons in three-flavor lattice QCD, Phys. Rev. Lett. 94 (2005) 011601 [hep-ph/0408306]. 
[3] I.F. Allison et al. [HPQCD and Fermilab Lattice Collaborations], Mass of the $B_{c}$ meson in three-flavor lattice QCD, Phys. Rev. Lett. 94 (2005) 172001 [hep-lat/0411027].

[4] C. Aubin et al. [Fermilab Lattice, MILC, and HPQCD Collaborations], Charmed meson decay constants in three-flavor lattice QCD, Phys. Rev. Lett. 95 (2005) 122002 [hep-lat/0506030].

[5] A.S. Kronfeld et al. [Fermilab Lattice, MILC, and HPQCD Collaborations], Predictions from lattice QCD, PoS LAT2005 (2006) 206 [Int. J. Mod. Phys. A 21 (2006) 713] [hep-lat/0509169];

A.S. Kronfeld, Predictions with lattice QCD, J. Phys. Conf. Ser. 46 (2006) 147 [hep-lat/0607011].

[6] Q. Mason et al. [HPQCD Collaboration], Accurate determinations of $\alpha_{s}$ from realistic lattice QCD, Phys. Rev. Lett. 95 (2005) 052002 [hep-lat/0503005].

[7] M. Creutz, Asymptotic-freedom scales, Phys. Rev. Lett. 45 (1980) 313.

[8] J. Bijnens, Quark mass dependence at two loops for meson properties, arXiv:0708.1377 [hep-lat].

[9] G.P. Lepage, Flavor-symmetry restoration and Symanzik improvement for staggered quarks, Phys. Rev. D 59 (1999) 074502 [hep-lat/9809157].

[10] C.W. Bernard et al. [MILC Collaboration], The QCD spectrum with three quark flavors, Phys. Rev. D 64 (2001) 054506 [hep-lat/0104002]; C. Aubin et al. [MILC Collaboration], Light hadrons with improved staggered quarks: approaching the continuum limit, Phys. Rev. D 70 (2004) 094505 [hep-lat/0402030].

[11] H.W. Hamber, E. Marinari, G. Parisi, and C. Rebbi, Numerical simulations of quantum chromodynamics, Phys. Lett. B 124 (1983) 99; F. Fucito and S. Solomon, The SU(3) deconfining transition in the presence of quarks, Phys. Lett. B 140 (1984) 387; S.A. Gottlieb, W. Liu, R.L. Renken, R.L. Sugar, and D. Toussaint, Hadron masses with two quark flavors, Phys. Rev. D 38 (1988) 2245.

[12] M. Creutz, Flavor extrapolations and staggered fermions, hep-lat/0603020 (revised in Ref. [14]).

[13] M. Creutz, Diseases with rooted staggered quarks, PoS LAT2006 (2006) 208 [hep-lat/0608020].

[14] M. Creutz, Chiral anomalies and rooted staggered fermions (originally called The evil that is rooting), Phys. Lett. B 649 (2007) 230 [hep-lat/0701018].

[15] M. Creutz, Reply to "Comment on 'Chiral anomalies and rooted staggered fermions'", Phys. Lett. B 649 (2007) 241 [arXiv:0704.2016 [hep-lat]].

[16] M. Creutz, Why rooting fails, these Proceedings [arXiv:0708.1295 [hep-lat]].

[17] C. Bernard, M. Golterman, Y. Shamir, and S.R. Sharpe, Comment on "Chiral anomalies and rooted staggered fermions”, Phys. Lett. B 649 (2007) 235 [hep-lat/0603027].

[18] E. Barberio et al. [Heavy Flavor Averaging Group], Averages of b-hadron properties at the end of 2006, arXiv:0704.3575 [hep-ex]; http://www.slac.stanford.edu/xorg/hfag/.

[19] Talks by P. Boyle, Y. Kuramashi, H. Matsufuru, and C. Urbach at this conference.

[20] M.A. Clark and A.D. Kennedy, Accelerating staggered fermion dynamics with the rational hybrid Monte Carlo (RHMC) algorithm, Phys. Rev. D 75 (2007) 011502 [hep-lat/0610047].

[21] L.H. Karsten and J. Smit, Lattice fermions: species doubling, chiral invariance, and the triangle anomaly, Nucl. Phys. B 183 (1981) 103.

[22] N. Kawamoto and J. Smit, Effective Lagrangian and dynamical symmetry breaking in strongly coupled lattice QCD, Nucl. Phys. B 192 (1981) 100. 
[23] H.S. Sharatchandra, H.J. Thun, and P. Weisz, Susskind fermions on a Euclidean lattice, Nucl. Phys. B 192 (1981) 205.

[24] L. Susskind, Lattice fermions, Phys. Rev. D 16 (1977) 3031.

[25] T. Banks, L. Susskind, and J.B. Kogut, Strong coupling calculations of lattice gauge theories: (1+1)-dimensional exercises, Phys. Rev. D 13 (1976) 1043.

[26] M.F.L. Golterman and J. Smit, Self-energy and flavor interpretation of staggered fermions, Nucl. Phys. B 245 (1984) 61.

[27] M.F.L. Golterman and J. Smit, Lattice baryons with staggered fermions, Nucl. Phys. B 255 (1985) 328.

[28] M.F.L. Golterman, Staggered mesons, Nucl. Phys. B 273 (1986) 663.

[29] M.F.L. Golterman, Irreducible representations of the staggered fermion symmetry group, Nucl. Phys. B 278 (1986) 417.

[30] G.W. Kilcup and S.R. Sharpe, A tool kit for staggered fermions, Nucl. Phys. B 283 (1987) 493.

[31] H. Joos and M. Schaefer, The representation theory of the symmetry group of lattice fermions as a basis for kinematics in lattice QCD, Z. Phys. C 34 (1987) 465.

[32] M. Wingate, J. Shigemitsu, C.T.H. Davies, G.P. Lepage, and H.D. Trottier, Heavy-light mesons with staggered light quarks, Phys. Rev. D 67 (2003) 054505 [hep-lat/0211014].

[33] F. Gliozzi, Spinor algebra of the one component lattice fermions, Nucl. Phys. B 204 (1982) 419.

[34] H. Kluberg-Stern, A. Morel, O. Napoly, and B. Petersson, Flavors of Lagrangian Susskind fermions, Nucl. Phys. B 220 (1983) 447.

[35] J.A. Bailey, Staggered baryon operators with flavor SU(3) quantum numbers, Phys. Rev. D 75 (2007) 114505 [hep-lat/0611023].

[36] S. Gottlieb, H. Na, and K. Nagata, Staggered diquarks for singly heavy baryons, arXiv:0707.3537 [hep-lat]; these Proceedings [arXiv:0710.0347 [hep-lat]]; H. Na and S. A. Gottlieb, Charm and bottom heavy-baryon mass spectrum from lattice QCD with 2+1 flavors, arXiv:0710.1422 [hep-lat].

[37] J. Smit and J.C. Vink, Renormalized Ward-Takahashi relations and topological susceptibility with staggered fermions, Nucl. Phys. B 298 (1988) 557.

[38] Q. Mason et al. [HPQCD Collaboration], High-precision determination of the light-quark masses from realistic lattice QCD, Phys. Rev. D 73 (2006) 114501 [hep-ph/0511160].

[39] E.B. Gregory, A.C. Irving, C.M. Richards, and C. McNeile, Methods for pseudoscalar flavor-singlet mesons with staggered fermions, arXiv:0709.4224 [hep-lat].

[40] C. Bernard et al. [MILC Collaboration], Update on the physics of light pseudoscalar mesons, PoS LAT2006 (2006) 163 [arXiv:hep-lat/0609053].

[41] W.-J. Lee and S.R. Sharpe, Partial flavor symmetry restoration for chiral staggered fermions, Phys. Rev. D 60 (1999) 114503 [hep-lat/9905023].

[42] C. Bernard, Chiral logs in the presence of staggered flavor symmetry breaking, Phys. Rev. D 65 (2002) 054031 [hep-lat/0111051].

[43] C. Aubin and C. Bernard, Pion and kaon masses in staggered chiral perturbation theory, Phys. Rev. D 68 (2003) 034014 [hep-lat/0304014]. 
[44] S.R. Sharpe and R.S. Van de Water, Staggered chiral perturbation theory at next-to-leading order, Phys. Rev. D 71 (2005) 114505 [hep-lat/0409018].

[45] T. Blum et al., Improving flavor symmetry in the Kogut-Susskind hadron spectrum, Phys. Rev. D 55 (1997) 1133 [hep-lat/9609036]; K. Orginos and D. Toussaint [MILC Collaboration], Testing improved actions for dynamical Kogut-Susskind quarks, Phys. Rev. D 59, 014501 (1999) [hep-lat/9805009]; J.F. Lagäe and D.K. Sinclair, Improved staggered quark actions with reduced flavor-symmetry violations for lattice QCD, Phys. Rev. D 59 (1999) 014511 [hep-lat/9806014].

[46] K. Orginos, D. Toussaint, and R.L. Sugar [MILC Collaboration], Variants of fattening and flavor-symmetry restoration, Phys. Rev. D 60 (1999) 054503 [hep-lat/9903032].

[47] C.W. Bernard et al. [MILC Collaboration], Scaling tests of the improved Kogut-Susskind quark action, Phys. Rev. D 61 (2000) 111502 [hep-lat/9912018].

[48] C. Aubin and C. Bernard, Pseudoscalar decay constants in staggered chiral perturbation theory, Phys. Rev. D 68 (2003) 074011 [hep-lat/0306026].

[49] S.R. Sharpe, Rooted staggered fermions: good, bad or ugly?, PoS LAT2006 (2006) 022 [hep-lat/0610094].

[50] C. Aubin et al. [MILC Collaboration], Light pseudoscalar decay constants, quark masses, and low energy constants from three-flavor lattice QCD, Phys. Rev. D 70 (2004) 114501 [hep-lat/0407028].

[51] J. Smit and J.C. Vink, Remnants of the index theorem on the lattice, Nucl. Phys. B 286 (1987) 485.

[52] A review oriented to lattice gauge theory is by P.H. Damgaard, The microscopic Dirac operator spectrum, Nucl. Phys. B Proc. Suppl. 106 (2002) 29 [hep-lat/0110192].

[53] J. Smit and J.C. Vink, Staggered fermions and topological susceptibility in lattice QCD at $\beta=5.7$ Phys. Lett. B 194 (1987) 433; J.C. Vink, Flavor symmetry breaking and zero-mode shift for staggered fermions, Phys. Lett. B 210 (1988) 211; Staggered fermions, topological charge and topological susceptibility in lattice QCD, Phys. Lett. B 212 (1988) 483.

[54] E. Follana, A. Hart, and C.T.H. Davies [HPQCD Collaboration], The index theorem and universality properties of the low-lying eigenvalues of improved staggered quarks, Phys. Rev. Lett. 93 (2004) 241601 [hep-lat/0406010]; S. Dür, C. Hoelbling, and U. Wenger, Staggered eigenvalue mimicry, Phys. Rev. D 70 (2004) 094502 [hep-lat/0406027].

[55] P.H. Damgaard, U.M. Heller, R. Niclasen, and K. Rummukainen, Staggered fermions and gauge field topology, Phys. Rev. D 61 (1999) 014501 [hep-lat/9907019].

[56] P.H. Damgaard, U.M. Heller, R. Niclasen, and B. Svetitsky, Patterns of spontaneous chiral symmetry breaking in vectorlike gauge theories, Nucl. Phys. B 633 (2002) 97 [hep-lat/0110028].

[57] K.Y. Wong and R.M. Woloshyn, Systematics of staggered fermion spectral properties and topology, Phys. Rev. D 71 (2005) 094508 [hep-lat/0412001]; E. Follana, A. Hart, C.T.H. Davies, and Q. Mason [HPQCD Collaboration], The low-lying Dirac spectrum of staggered quarks, Phys. Rev. D 72 (2005) 054501 [hep-lat/0507011].

[58] M. Golterman, Y. Shamir, and B. Svetitsky, Breakdown of staggered fermions at nonzero chemical potential, Phys. Rev. D 74 (2006) 071501 [hep-lat/0602026].

[59] An efficient derivation follows from the exposition of A. Chodos and G. Gallatin, Generalized Goldstone theorem, J. Math. Phys. 42 (2001) 3282 [math-ph/0008037].

[60] C. Itzykson and J.B. Zuber, Quantum Field Theory, McGraw-Hill, New York (1980), p. 520. 
[61] B. Bunk, M. Della Morte, K. Jansen, and F. Knechtli, Locality with staggered fermions, Nucl. Phys. B 697 (2004) 343 [hep-lat/0403022]; A. Hart and E. Müller, The locality of the square-root method for improved staggered quarks, Phys. Rev. D 70 (2004) 057502 [hep-lat/0406030].

[62] D.H. Adams, On the fourth root prescription for dynamical staggered fermions, Phys. Rev. D 72 (2005) 114512 [hep-lat/0411030].

[63] F. Fucito, E. Marinari, G. Parisi, and C. Rebbi, A proposal for Monte Carlo simulations of fermionic systems, Nucl. Phys. B 180 (1981) 369; G.G. Batrouni et al., Langevin simulations of lattice field theories, Phys. Rev. D 32 (1985) 2736; S.A. Gottlieb et al., Hybrid molecular dynamics algorithms for the numerical simulation of quantum chromodynamics, Phys. Rev. D 35 (1987) 2531.

[64] C. Bernard, Staggered chiral perturbation theory and the fourth-root trick, Phys. Rev. D 73 (2006) 114503 [hep-lat/0603011].

[65] C. Bernard et al., Status of the MILC light pseudoscalar meson project, arXiv:0710.1118 [hep-lat].

[66] W.A. Bardeen and J.N. Simone, private communication (2004).

[67] B. Billeter, C. DeTar and J. Osborn, Topological susceptibility in staggered fermion chiral perturbation theory, Phys. Rev. D 70 (2004) 077502 [hep-lat/0406032]; C. Bernard et al., The 2+1 flavor topological susceptibility from the Asqtad action at $0.06 \mathrm{fm}$, arXiv:0710.3124 [hep-lat].

[68] S. Prelovšek, Effects of staggered fermions and mixed actions on the scalar correlator, Phys. Rev. D 73 (2006) 014506 [hep-lat/0510080].

[69] W.A. Bardeen, A. Duncan, E. Eichten, N. Isgur, and H. Thacker, Chiral loops and ghost states in the quenched scalar propagator, Phys. Rev. D 65 (2002) 014509 [hep-lat/0106008]; W.A. Bardeen, E. Eichten, and H. Thacker, Chiral Lagrangian parameters for scalar and pseudoscalar mesons, Phys. Rev. D 69 (2004) 054502 [hep-lat/0307023].

[70] C. Bernard, C. DeTar, Z. Fu, and S. Prelovšek, Scalar meson spectroscopy with lattice staggered fermions, arXiv:0707.2402 [hep-lat]; Taste breaking effects in scalar meson correlators, PoS LAT2006 (2006) 173 [hep-lat/0610031].

[71] C. Aubin, J. Laiho, and R.S. Van de Water, Taste violations in the scalar correlator in mixed action simulations, PoS LAT2007 (2007) 88 [arXiv:0710.0804 [hep-lat]].

[72] K. Jansen, Actions for dynamical fermion simulations: are we ready to go?, Nucl. Phys. B Proc. Suppl. 129 (2004) 3 [hep-lat/0311039].

[73] C. Bernard, M. Golterman, and Y. Shamir, Observations on staggered fermions at non-zero lattice spacing, Phys. Rev. D 73 (2006) 114511 [hep-lat/0604017].

[74] D.H. Adams, A simplified test of universality in lattice QCD, Phys. Rev. Lett. 92 (2004) 162002 [hep-lat/0312025]; Testing universality and the fractional power prescription for the staggered fermion determinant, Nucl. Phys. B Proc. Suppl. 140 (2005) 148 [hep-lat/0409013].

[75] T. Reisz, A convergence theorem for lattice Feynman integrals with massless propagators, Commun. Math. Phys. 116 (1988) 573; A power counting theorem for Feynman integrals on the lattice, Commun. Math. Phys. 116 (1988) 81; Renormalization of Feynman integrals on the lattice, Commun. Math. Phys. 117 (1988) 79; Renormalization of lattice Feynman integrals with massless propagators, Commun. Math. Phys. 117 (1988) 639; Lattice gauge theory: renormalization to all orders in the loop expansion, Nucl. Phys. B 318 (1989) 417.

[76] J. Giedt, Power-counting theorem for staggered fermions, Nucl. Phys. B 782 (2007) 134 [hep-lat/0606003]. 
[77] C. Bernard, M. Golterman, and Y. Shamir, Effective field theories for rooted staggered fermions, these Proceedings [arXiv:0709.2180 [hep-lat]].

[78] Y. Shamir, Locality of the fourth root of the staggered-fermion determinant: renormalization-group approach, Phys. Rev. D 71 (2005) 034509 [hep-lat/0412014]; Renormalization-group analysis of the validity of staggered-fermion QCD with the fourth-root recipe, Phys. Rev. D 75 (2007) 054503 [hep-lat/0607007].

[79] C. Bernard et al., The locality of the fourth root of staggered fermion determinant in the interacting case, PoS LAT2005 (2006) 114 [hep-lat/0509176].

[80] I learned this argument from Peter Hasenfratz in 1992. This summer I asked him if he knew a nice written reference, and he replied "I do not remember reading anything written about [the subtlety]; perhaps people consider this too trivial."

[81] S. Dürr and C. Hoelbling, Staggered versus overlap fermions: a study in the Schwinger model with $N_{f}=0,1,2$, Phys. Rev. D 69 (2004) 034503 [hep-lat/0311002]; S. Dürr and C. Hoelbling, Scaling tests with dynamical overlap and rooted staggered fermions, Phys. Rev. D 71 (2005) 054501 [hep-lat/0411022].

[82] C. Bernard, Order of the chiral and continuum limits in staggered chiral perturbation theory, Phys. Rev. D 71 (2005) 094020 [hep-lat/0412030].

[83] C. Bernard, M. Golterman, Y. Shamir, and S.R. Sharpe, 't Hooft vertices, partial quenching, and rooted staggered QCD, arXiv:0711.0696 [hep-lat].

[84] S. Naik, On-shell improved lattice action for QCD with Susskind fermions and asymptotic-freedom scale, Nucl. Phys. B 316 (1989) 238.

[85] E. Follana et al. [HPQCD Collaboration], Highly-improved staggered quarks on the lattice, with applications to charm physics, Phys. Rev. D 75 (2007) 054502 [hep-lat/0610092].

[86] A. Hasenfratz and F. Knechtli, Flavor symmetry and the static potential with hypercubic blocking, Phys. Rev. D 64 (2001) 034504 [hep-lat/0103029].

[87] K.Y. Wong and R.M. Woloshyn, Dynamical simulations with highly improved staggered quarks, arXiv:0710.0737 [hep-lat].

[88] P. Weisz, Continuum limit improved lattice action for pure Yang-Mills theory 1, Nucl. Phys. B 212 (1983) 1; P. Weisz and R. Wohlert, Continuum limit improved lattice action for pure Yang-Mills theory 2, Nucl. Phys. B 236 (1984) 397 [Erratum ibid. 247 (1984) 544]; G. Curci, P. Menotti, and G. Paffuti, Symanzik's improved Lagrangian for lattice gauge theory, Phys. Lett. B 130 (1983) 205 [Erratum ibid. 135 (1984) 516]; M. Lüscher and P. Weisz, On-shell improved lattice gauge theories, Commun. Math. Phys. 97 (1985) 59 [Erratum ibid. 98 (1985) 433].

[89] M. Lüscher and P. Weisz, Computation of the action for on-shell improved lattice gauge theories at weak coupling, Phys. Lett. B 158 (1985) 250.

[90] Z. Hao, G.M. von Hippel, R.R. Horgan, Q.J. Mason, and H.D. Trottier, Unquenching effects on the coefficients of the Lüscher-Weisz action, Phys. Rev. D 76 (2007) 034507 [arXiv:0705.4660 [hep-lat]]; these Proceedings [arXiv:0708.2404 [hep-lat]].

[91] A. Hart and G.M. von Hippel, private communication (2007).

[92] D. Toussaint, private communication (2007).

[93] S. Weinberg, Phenomenological Lagrangians, Physica A 96 (1979) 327.

[94] C. Bernard, private communication (2007). 